\section{Isolamento e identificação da microbiota fúngica e de dermatófitos da pele de eqüinos hígidos e daqueles afetados por dermatofitose*}

\author{
Isolation and identification of fungal microbiota and of \\ dermatophytes irom healthy horses and from dermatophytosis \\ -affected horses
}

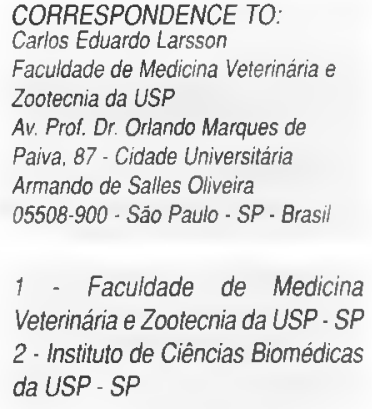

CE TO

Faculdade de Medicina Veterinária Zootecnia da USP

Prot. Dr. Orlando Marques de

Armando de Salles Oliveira

05508-900 - Säo Paulo - SP - Bras

Veterinária e Zootecnia da USP - SP da USP - SP

\author{
Marcia Mayumi ISHIKAWA '; Ronaldo LUCAS '; Carlos Eduardo LARSSON '; \\ Walderez (;AMBALE '; Wilson Roberto FERNANDES'
}

\title{
RESUMO
}

Pela inexistência na literatura latino-americana e brasileira de trabalhos que enfoquem a microbiota fúngica de eqüinos hígidos e as principais espécies de dermatófitos em casos de eqüinos com lesões sugestivas de infecção dermatofítica do tegumento cutâneo, utilizaram-se 175 eqüinos, de ambos os sexos, tanto de raça definida como daqueles sem perfeita definição racial, de diferentes idades e que foram reunidos em 2 grupos. O GRUPO I composto de 133 eqüinos assintomáticos e desprovidos de lesões cutâneas, dos quais, após exame dermatológico, interposição da Luz de Wood (48 eqüinos), colheram-se, pela técnica do carpete, material que foi semeado em meios de ágar Sabouraud Dextrose, Mycobiotic ágar, Tricophyton ágar 3, Tricophyton ágar 5 e incubados a $25^{\circ}$ e $37^{\circ} \mathrm{C}$ durante 30 dias. Isolaram-se: Penicillium sp (80,4\%), Rhizopus sp (62,4\%), Aspergillus sp (41,3\%), Fusarium sp $(40,6 \%)$, Cladosporium sp (33,1\%), Trichoderma sp $(21,0 \%)$, Mucor sp (18,0\%), Epicoccum $s p(12,0 \%)$, Mycelia sterillia $(8,8 \%)$, Rhodotorula sp (7,5\%), Neurospora sp (4,5\%), Alternaria sp (3,7\%), Aureobasidium sp (3,7\%), Geotrichum sp $(3,0 \%)$, Paecilomyces sp $(2,2 \%)$, Monascus $s p(2,2 \%)$, Cephalosporium $s p(1,5 \%)$, Nigrospora $s p(0,7 \%)$, Scopulariopsis brevicaulis $(0,7 \%)$, Trichosporon sp $(0,7 \%)$. O GRUPO II foi composto por 42 eqüinos portadores de lesões sugestivas de dermatofitose que, após terem sido submetidos a exame dermatológico, expostos à luz de Wood (22 eqüinos), tiveram pelame e crostas submetidos a cultivo micológico, isolando-se em $6(14,3 \%)$ cepas de Dermatophylus congolensis e em $3(7,1 \%)$ eqüinos houve o crescimento de dermatófitos da espécie Microsporum canis. Dos 70 eqüinos expostos à radiação ultravioleta observou-se falsa fluorescência em 2 animais.

UNITERMOS: Dermatồfitos; Equidae.

\section{INTRODUÇĀO}

$\mathrm{O}$ s levantamentos sobre a microbiota normal dos grandes animais ané entĩo realizados têm sido estritamente qualitativos (Scott 1958 ) e demonstram que a pele e o pelame de herbívoros mono e poligástricos são verdadeiras fontes de fungos e bactérias. Dentre os fungos já isolados da superfície corpórea de eqüinos criados em países curopeus e dal Américil do Norte arrolam-se: Absidia spp. Acremonium spp, Alternaria spp, Aspergillus spp. Aureohasidium spp, Candida spp, Cephalosporium spp. Chactomium spp, Cladosporium spp, Cryptococcus spp. Diplosporum spp, Fusarium spp, Geomyces spp, Helmintosporum spp, Homodendrum spp, Mucor spp, Nigrospora spp, Penicilium sp, Phoma sp.

\footnotetext{
* 'Trabalho execulide sob os aluspícios da Fundaçĩo de Amparo à Pesquisa do Estido de

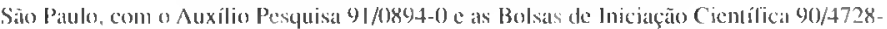
5 c 92/1265-0. e apresentado em sessīo plenária do $15^{\circ}$ Congresso Pan-Americano de Ciências Veterinárias, na Cidade do Méxica (DF) em outubro de 1994.
}

As condições climáticass, geográficàs e socioeconômicàs brasileiras propiciam a ocorrencia de inúmeras micoses profundas e superficiais tanto no homem como nos animais. Enfocando as micoses superficiais, extremamente freqüentes nos animais domésticos, ou em certos grupos profissionais e em donos contactantes, alguns pesquisadores brasileiros têm relatado a ocorrência de surtos esporádicos ou descrito a magnitude de sua ocorrência em serviços, clínicas particulares, vilas hípicals etc. (Londero22. 1963 ; Fischman ${ }^{16}$, 1966; Londero et al.24, 197(); Proença: Assumpção ${ }^{37}$, 1979; Gambale et al. 17, 1987). Estes incuéritos e relatos de prevalência de dermatófitos têm, mais freqüentemente, abrangido as micoses superficiais dos carnívoros domésticos pela sua estreita relação de convivência com o homem (Proença; Assumpção. ${ }^{37}$, 1979; Gambale et al. $\left.{ }^{17}, 1987\right)$. Todavia, praticamente nada está assentado no Brasil no tocante à microbiota fúngica, normal e patogênica, de outros espécimes, a exemplo dos eqüinos, tanto os de 


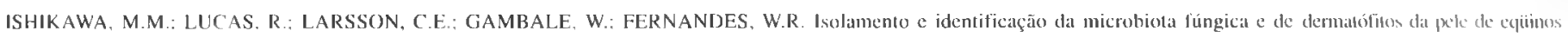
hígidos e dacueles atlelidos por dermattofitose. Braz. J. vet. Res. anim. Sei. São Paulo, v.33, n. 3. p.170-175. 1996.

tração como aqueles de esporte e lazer. Raros são os trabalhos nacionais (Londero'22, 1963; Fischman16, 1966) indexados envolvendo o lema. Sabe-se que as dermatofitoses dos eqüinos constituem ergodermatoses e antropozoonoses que acometem com frequiência cavalariços, jóqueis, veterinários, treinadores, amazonas etc. Nos prados e vilas hípicas são freqüentes as descrições do chamado "prurido do jóquei", modalidade de linha crural devida ao $M$. equimmm.

Sobre equírleos nada se compulsa da bibliogralia nacional no atinente à microbiota fúngica de animais hígidos. O conhecimento desta microbiota constitui o primeiro passo para o estabelecimento do diagnóstico etiológico e da terapia mais adequada para dermatopatias de origem lúngica.

No que tange às espécies de fungos patogênicos para equinos, Sco11 ${ }^{+6}(1988)$ arrolou o $M$. gypseam, T. equimum, $T$. mentagrophytes, T. verrucosum, $M$. equimum e $M$. canis. Destes, encontram-se apenas citaçoes, na bibliografia brasileira, da ocorrência de tricofitose (pelo T. verrucosum) e de microsporíase (pelo $M$. canis), publicados pelos gaúchos Londero et al.25 (1967) e Fischman'6 (1966).

O delineamento da microbiota fúngica da pele e do pelame de animais hígidos 1 em sido realizado, em nossas condições, pelas técnicas adaptadas de Mariat; Tapia2 (1966) e Mariat; Adan-Campos ${ }^{27}$ (1967), que consistem, basicamente, na esfregadura de fragmentos de carpetes estéreis sobre a pele dos animais em estudo, decalcando, então, em meios de cultivo específicos.

O diagnóstico das dermatolitoses dos eqüinos, similarmente ao descrito para as demais espécies, pode ser executado pela inspesáno indireta do pelame e/ou das lesões utilizando-se da radiação UV (Lâmpada de Wood); exame microscópico direto do pelame e de crostas clarificadas pelo $\mathrm{KOH}(10 \%)$; e preferencial e eletivamente, pelo cultive em meios especificos (Saboraud, Mycosel), acrescidos de impedientes de crescimento de contaminantes e suplementados com niacina (T-5), tiamina e inositol (T-3), e em distintas temperalluras na dependência da espécie fúngica em suspeição. Em caso de crescimento de dermatófitos procede-se à identificação laxonômica macro e microscópica pelos mélodos de Rapper; Fewell38 (196.5); Arx $^{3}$ (1970); Lodder21 (1970); Rebell; Taplin ${ }^{39}$ (1970); Barron5 (1971); McGinnis20 (1980); Kreger-Von Rij26 (1984).

Levando em consideração os latos expostos, delineou-se o presente trabalho tendo como objetivos: determinar a microbiotal lúngical na pele e pelame de eqüinos hígidos criados em São Paulo; determinar as espécies de dermatófïlos palogênicos, por meio do cultivo micológico, de material provindo de eqüinos com lesões sugestivas de infecção fúngica; determinar a praticabilidade, sensibilidade, especificidade e concordância da radiação ultravioleta (Lâmpada de Wood), previamente interposta no pelame de animais hígi- dos e daqueles com lesões dermatofílicas, com relação aos resultados obtidos no exame micológico do material semeado.

\section{MATERIAL E MÉTODO}

Utilizaram-se de 175 eqüinos, $98(56,(0 \%)$ machos e 77 (44,0\%) fêmeas, 123 de raça definida (American Trotler, Anglo Argentino, Árabe, Brasileiro de Hipismo, Mangalarga, Puro Sangue Inglês, Quarto de Milha, Sela Argentina, Andaluz e Appaloosa), como de 52 animais sem perfeita definição racial (SRD).

Quanto à distribuição etária, 28 (16,0\%) tinham menos de I ano de idade.

Esta amostragem foi dividida em dois grupos, a saber:

GRUPO I - Constituído de 133 eqüinos assintomálicos e desprovidos de lesões cutâneas sugestivas de dermatolitose, $77(58,0 \%)$ machos e $56(42,0 \%)$ lềmeas, $103(77,5 \%)$ com raça definida (CRD) e $30(22,5 \%)$ sem perfeita definição racial (SRD), agrupados nas faixas etárias de menos de 1 ano de idade (25-18,8\%), 1 a 3 anos (23-17,3\%), de 3 a 5 anos (33-24,8\%), de 5 a 7 anos $(21-15,8 \%)$, de 7 a 9 anos (13$9,8 \%$ ) e com mais de 9 anos (18-13,5\%). Utilizou-se esto grupo para a determinação da microbiota fúngica indígena.

Nestes animais, após prévia identificação, exames físico e dermatológico, interpôs-se a Lâmpada de Wood em 68 animais e a seguir colheu-se pelo método de Mariat: AdanCampos $^{27}$ (1967) material para a semeadura nos meios específicos. Para tanto, os retalhos estéreis de carpete foram friccionados em faixas paralelas, em ambas as metades longitudinais do corpo, iniciando-se pela região da lace, progredindo pelas regiões cervical, escapular, torácica, lombar, flancos, ilíaca e das ancas. Para cada animal utilizaram-se cinco retalhos de carpete, que após a colheita foram reembrulhados em seus próprios envoltórios aluminizados estéreis e levados para semeadura, feita em laboratório, num período máximo de 4 horas, a contar do momento dal colheila.

GRUPO 11 - Constituído de 42 animais portadores do lesões sugestivas de dermatofitoses, atendidos "in loce" no Serviço de Ambulatório e Hospitalização de Grandes Animais (Setor Eqüinos) das Disciplinas VCM 303 e 50), no Hospital da FMVZ-USP ou em baias de vilas hípicals ou de propriedades visitadas.

Destes 42 animais, $21(50 \%)$ eram machos e $21(50 \%)$ eram lềmeas, $22(52,4 \%)$ com raça definida e $20(47,6 \%)$ sem perfeita definição racial. Agrupados nas faixas etárias com menos de 1 ano $(3-7,1 \%)$, de 1 a 3 anos $(8-19,0 \%)$, de 3 a 5 anos $(12-28,6 \%)$, de 5 a 7 anos $(8-19,0 \%)$, de 7 a 9 anos (6-14,3\%) e com mais de 9 anos $(5-12,0 \%)$.

Após prévia identificação, anamnese, exames físico e dermatológico, procedeu-se de início à interposição da Luz de 


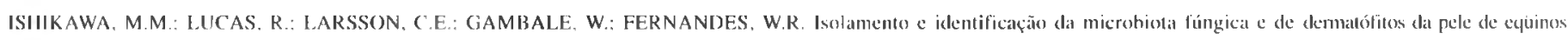
hígidon e dacuedes afelados por dermatolitose. Bray. J. vet. Res. anim. Sci. São Paulo, v.33, n..3. p. 170)-175, 1996.

Wood (em 22 animais) e a seguir à colheita de material (pelame, escamas e crostas) das lesões. O material colhido foi transportado em placas de Petri estéreis, ao laboratório, para num período máximo de 4 horas ser semeado em meios próprios de cultivo fúngico.

Para os animais dos dois grupos, utilizaram-se os meios de Sabouraud Dextrose ágar acrescido de cloranfenicol (DIFCO), Mycobiotic ágar (DIFCO), Tricophyton ágar 3 (DIFCO) (específico para cepas de T. verrucosum, que necessilam de tiamina e inositol), Tricophyton ágar 5 (DIFCO) (específico para a espécie $T$. equimum, que requer ácido nicotínico), sendo as culturas realizadas em duplicata e, respectivamente, incubadas a $25^{\circ} \mathrm{C}$, e também a $37^{\circ} \mathrm{C}$, no caso do Tricophyton 3.

As placas foram mantidas incubadas por 30 dias para excluir possíveis falsos negativos. Quando do crescimento fúngico, procedeu-se à identificação taxonômica pelos métodos preconizados por Rapper; Fewell ${ }^{38}$ (1965); Arx $^{3}$ (1970); Lodder21 (1970); Rebell; Taplin39 (1970); Barron5 (1971); McGinnis26 (1980); Kreger-Von Rij' ${ }^{19}$ (1984).

\section{RESULTADOS}

Os resultados obtidos com a amostragem em tela foram:

(;RUPO I - Nos equiinos hígidos examinados procedeuse à identificação dos gêneros de fungos pertencentes à microbiota indígena.

Houve crescimento de Penicillium sp em $80,4 \%$ das culluras, Rhizopus sp em 62,4\%, Aspergillus sp em 41,3\%, Fusarium sp em 40,6\%, Cladosporium sp em 33,1\%, Trichoderma sp em $21,0 \%$, Mucor sp em 18,0\%, Epicoc(um sp) em 12,0\%, Mycelia sterillia em 8,8\%, Rhodotorula sp em $7,5 \%$, Neurospora sp em 4,5\%, Alternaria e Aureobasidium $s p$, ambos em 3,7\%, Geotrichum sp em 3,0\%, Monascus e Pacrilonuces, ambos em 3,7\%, Circinella e Cephaslosporium sp, os dois gêneros, em $1,5 \% \mathrm{e}$, finalmente, os três gêneros restantes, Nigrospora, Scopulariopsis e Trichosporon sp, em $0,7 \%$ dos cultivos.

Não houve crescimento de nenhuma espécie de dermatófito nas culturas dos 133 animais hígidos.

(IRUPO II - Dos 42 eqüinos portadores de lesões supostamente de etiologia dermatofítica, verificou-se que na realidade $6(14,3 \%)$ animais apresentavam quadro dermatológico compatível com infecção bacteriana pelo gênero Dermatophilus sp. Após a colheita de material para exames, não só micológico, mas também bacteriológico, pôde-se confirmar o diagnóstico presuntivo de piodermite superficial pelo Dermatophilus congolensis.

Em apenas $3(7,1 \%)$ animais, 2 machos com, respectivamente, 3 e 5 anos de idade, da raça Mangalarga, e 1 fêmea, Puro Sangue Inglês, com 3 anos, houve o cresci- mento de dermatófitos da espécie Microsporum canis. Nos demais $33(78.6 \%)$, não houve crescimento de nenhuma espécie de dermatófito.

Relativamente à inspeção indirela utilizando-se da luz ultravioleta na faixa de $2500 \mathrm{~A}^{0}$ (Luz de Wood), realizada em 70 animais, sendo $48(68,5 \%)$ pertencentes ao GRUPO 1 (eqüinos assintomáticos), e $22(31.5 \%)$ daqueles do GRUPO II (equiinos com lesões), não houve lluorescência em nenhum dos animais do GRUPO I. Detectou-se positividade em 2 dos animais com lesões cutâneas (GRUPO II), lodavia os cultivos micológicos posteriores não se revelaram positivos quanto ao crescimento de dermatófitos.

\section{DISCUSSĀO}

Os resultados obtidos nos constituintes do GRUPO I não podem ser cotejados pela inexistência de trabalhos similares na América Latina. Entretanto, tal cotejamento é possível com levantamentos realizados nos EUA (Scott: BetinckSmith ${ }^{44}, 1985:$ Scott $^{46}, 1988$ ) e na Finlândia (Aho', 1983). Neste último, houve a preocupação de quantificar numericámente os fungos mais freqüentes; assim se pôde constatar, a despeito de Aho' (1983) ter usado meios de cultivo diferentes dos aqui utilizados, que os gêneros Penicillium. Aspergillus, Mucor e Fusarium são os mais comuns em pelames de equiinos hígidos, mesmo em se tratando de países tão distintos, climática e geograficamente. As diferenças, ao se considerar os cinco gêneros mais frequientes, dizem respeito a Rhizopus e Cladosporium (Brasil) e Trichothecium (Finlândià).

Quanto ao cotejamento com levantamentos dispostos na bibliografia americana (Scott; Betinck-Smith ${ }^{+14}$, 1985; Scott $^{+6}, 1988$ ), verifica-se que houve coincidência de isolamentos de fungos no Brásil e nos EUA, dos gêneros Alternaria, Aspergillas, Cephalosporium, Penicillium. Fusarium, Cladosporium, Mucor; Rhodotorula, Rhizopus e Trichoderma. Neste trabalho, provavelmente a microbiolat fúngica isolada reflete a abundância de esporos de fungos no ambiente criatório (provindo do solo, feno, grãos de cereais) e que, uma vez at ingindo o revestimento cutâneo dos equiinos, foram ali mantidos pela eletricidade estálica originária do roçar de fômites (rascadeiras, escovas, mantas, arreios, etc.) sobre o pelame (Euzeby et al. ${ }^{15}, 1973$ : Aho', 1983).

Ylimaki49 (1981), examinando a microflora em grãos e demais ingredientes de raçoes, na Finlândia, observou que Alternaria, Cladosporium, Fusarium, Penicillum são os fungos mais comuns em amostras de grãos e que Penicillum. Mucor e Rhizopus o são em amostras de ingredientes de ração compostos de grãos, misturas alimentares comerciais, feno e silagem.

Quanto aos gêneros ora isolados, Aho' (1983) afirmou 
ISHIKAWA. M.M.: LUCAS. R.; LARSSON, C.E.; GAMBALE, W.: FERNANDES, W.R. Isolamento e identificação da microbiola fúngical e de dermalófitos dal pele de ęüinos hígidos e dilqueles afetados por dermalofitose. Braz. J. vet. Res, anim. Sei. São Paulo, v.33, п.,3, p. 170)-175, 1996.

que o Macor é mais freqüente em bovinos e que Cladosporium e Penicillium são mais encontrados em cães, relativamente aos eqüinos.

No que tange ao papel patogênico dos fungos ditos saprófitas, isolados, dentre os três gêneros mais freqüentes (Penicillium, Rhizopus e Aspergillus) no presente levantamento, verifica-se que o Aspergillus sp pode, em condições experimentais, desintegrar o córtex de pêlos oriundos de bovinos e humanos (English ${ }^{14}, 1965$ ). Este mesmo gênero já foi isolado de granulomas subcutâneos de bovinos (Davis; Schaeferl2, 1962), e de dermatite papular em suínos (Tabuchit7, 1963).

Dos fungos ditos constituintes da microbiota indígena ora isolados (GRUPO I), apenas os gêneros Geotrichum sp e Alternaria sp têm sido incriminados como agentes etiológicos de micoses de equiinos (Santos et al. ${ }^{43}$, 1983; Scott $^{46}$, 1988).

A espécie Geotrichum candidum é um agente micótico cosmopolita que tem sido isolado de frutos, legumes, produtos lácteos e do solo. A geotricose em Medicina Veterinária já foi assinalada em suínos (Morquer et al.29, 1955), bovinos (Ainswort: Austwick², 1955), cães (Lincon; Adcock20, 1968) e equiinos (Santos et al.42, 43, 1972, 1983; Santos; Camargo ${ }^{40}$, 1975; Mos et al. ${ }^{30}$, 1978; Chengappa et al. ${ }^{10}$, 1984). Segundo Santos et al. ${ }^{43}$ (1983), a geotricose no Brasil tem sido assinalada, mormente em equiinos destinados a práticas esportivas, com periódica assistência veterinária e com excelentes trato e manejo. Clinicamente, nos eqüinos, manifestase sob a forma de áreas extensas de alopecia, descamação, hiperidrose e prurido discretos. O quadro lesional observado em 6 animais, por aqueles autores, foi controlado em 2 semanas de utilização tópica de solução de violeta de genciana a $2 \%, 2$ vezes ao dia.

Nos quatro casos em que ora se obteve êxito no isolamento de Geotrichum sp, os eqüinos não manifestavam nenhuma lesão cutânea cuando da colheita. Todavia, como ressaltaram Santos et al.43 (1983), a utilização de antibióti$\cos$ e de quimioterápicos potentes no controle de processos infecciosos pode facilmente favorecer a atividade de agentes oportunistas, a exemplo do Geotrichum sp, conferindo-lhes então a possibilidade de exibir suas potencialidades patogênicas.

Entre as espécies do gênero Alternaria, comumente saprofíticas, a chamada Alternaria tenuis já foi isolada de lesões papulonodulares disseminadas pela cabeça, tórax e membros de eqüinos.

Tais lesões histopatologicamente se caracterizaram por uma dermatite piogranulomatosa, plena de hifas septadas e de clamidosporos de Alternaria tenuis (Coles"1, 1978).

Este mesmo gênero tem sido identificado em lesões cutâneas de equiinos, sem contudo ter havido a necessária comprovação histopatológica (Euzeby et al.15, 1973;
Percebois et al.33, 1978). Euzeby et al. ${ }^{15}$ (1973), em trabalho levado a cabo em Lyon, na França, abordando os saprófitas, por eles denominados de pseudodermatófitos, afirmaram que o gênero Alternaria potencialmente pode proliferar em lesões escoriativas primárias de pele, produzindo filamentos que modificam a lesão inicial dando-lhes características clínicas de uma lesão típica de tinha. Afirmaram, ainda, que mesmo peles normais podem se tornar suscetíveis a infecções fúngicas pelas aplicações tópicas intempestivas de corticóides e de antibióticos, que favoreciam todo o potencial patogênico dos fungos ditos "inofensivos" para uma pele normal.

Em 42 eqüinos constituintes do GRUPO II, quais sejam. animais com lesões compatíveis com dermatofitose, puderamse na realidade isolar, em $6(14,3 \%)$ destes, cepas de Dermatophilus congolensis. O primeiro relato de dermatofilose equiina na América do Sul data de 1965, tendo sido assinalado na Argentina, por Perez-Catan; Di Roceo ${ }^{34}$ (1965), em animais provindos dos EUA. No Brasil, a primeira descrição realizada, por Londero et al. ${ }^{25}$, em 1967. no Rio Grande do Sul, refere-se a um potro crioulo que veio a óbito devido à gravidade do quadro. Londero ${ }^{23}$ ( 1976 ) considera que os dados referentes à prevalência e aos rellexos econômicos decorrentes da dermatofitose são esparsos na bibliografia brasileira; contudo, destaca que a infecção está amplamente disseminada em eqüinos criados no Rio Grande do Sul. Em Minas Gerais, a primeira descrição em eqüinos coube a Barbosa et al. ${ }^{4}$ (1970). No Estado de São Paulo, a dermatofitose foi assinalada pela primeira vez por Portugal; Fischman ${ }^{35}$ (1974). Cinco anos mais tarde, Portugal et al. ${ }^{36}$ (1979) relataram três surtos, nos municípios de Presidente Prudente, Tatuí e Martinópolis, envolvendo 7 eqüinos adultos (3 PSI e 4 Quartos de Milha).

No cotidiano da clínica de eqüinos, com frequiência diagnosticam-se casos de processos inflamatórios do folículo piloso ou com acometimento da derme e do tecido subcutâneo adjacentes, processos estes que são denominados, respectivamente, de foliculite e furunculose. A etiopatogenia destes quadros é multifatorial, incluindo bactérias, mormente Dermatophilus congolesis e Staphylococcus atreus, fungos (Trichophyton e Microsporum sp) e parasitas (Demoder equi e Pelodera strongy/oides). Como fatores predisponentes arrolam-se: traumas mecânicos, devidos, primária ou secundariamente, a dípleros, calor e umidade e, finalmente, condições de manejo deficientes (Scott"4, 1985). Seis casos no presente levantamento referem-se, lambém, a eqüinos adultos, alojados em cocheiras com parcas condições higiênicas, confirmando-se a possibilidade de transmissão por meio de vetores animados ou por meio de fômites mal higienizados.

Com referência aos animais do GRUPO II, não se logrou êxito em obter um número relativamente grande de equiinos,

\section{SERVIÇO DE BIBLIOTECA E DOCUMENTAÇĀO \\ FACULDADE DE MEDICINA VETERINARIA. E ZOOTECNIA DA USP}




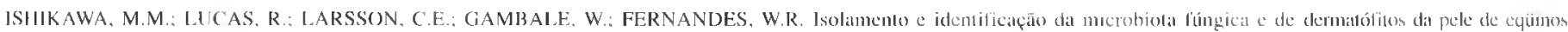

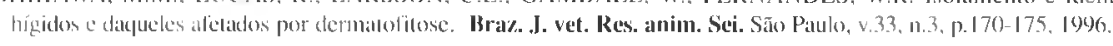

a despeito das tentativas junto a clínicos autônomos e em serviços veterinários de vilas hípicas. Provavelmente, muitos dos casos de dermatofitoses além de sua autolimitação são precocemente submetidos à terapia por tratadores, impedindo, assim, a colheita do material previamente à interposição de condula medicamentosa, tal como freqüientemente se observou ao longo do período de elaboração do presente levantamento.

Nos isolamentos oblidos, pôde-se identificar e sistematizar o agente como da espécic Microsporum canis

Entre os dermatófitos zoofílicos, o $M$. canis tem sido relatado na literatura estrangeira desde 1896 (Bodin', 1986), com frequiências de isolamento variando de $1,26 \%$ a $72.5 \%$ (Broce-Rousseau ef al.7, 1927; Carter et al.", 1970); Takatori et al. ${ }^{48}, 1981$ ). A despeito de uma aparente alta prevalência de infecção, em tempos idos, na década de 60, tal modalidade de microsporíase era considerada rara (Fischman ${ }^{16}$, 1966). O primeiro relato brasileiro de infecção eqüina por $M$. canis coube a Fischmanto (1966), que o descreveu em um eqüino de 7 anos, criado na área suburbana de Santa Maria (RS), em contato com cães, gatos e crianças, que posteriormente desenvolveram lesões de tinha por $M$. canis.

Ainda no Brasil, Santos et al. ${ }^{+1}$ (1992) em investigação ainda não publicada, realizada com 161 equiinos, no período 1969-1988, com lesões sugestivas de dermatofitose, isolaram em 4 cultivos (25\%) 3 cepas de Microsporum equinum e 1 do gênero Microsporum sp que, infelizmente, não foi identificatda quanto à espécie, nãa se afastando, portanto, a possibilidade de ser também $M$. c'anis.

No que tange aos resultados da utilização da radiação ultravioleta na inspeção indireta, tanto em animais desprovidos de lesões culâneas como naqueles portadores de quadro lesional, compatível com dermatofitose, em que pese o número reduzido de animais (48 do GRUPO) I e 22 do GRUPO II) em que se lançou mão deste recurso de diagnóstico, não se detectou fluorescência em nenhum dos casos. Sabe-se que a chamada Luz de Wood demonstra fluorescência em infecções fúngicas "in vivo" decorrentes da presença de M. canis, M. andonini, T. schoenleinii e M. equinum, graças à presença de metabólitos do triptofano produzidos por estes fungos (Scott ${ }^{45}, 46,1985,1988$ ). Infelizmente, o exame pela Luz de Wood raramente é positivo quando da interposição) em lesões que acometem os chamados grandes animais. No presente levantamento obteve-se crescimento apenas em 3 casos de dermatófito da espécie $M$. camis, que lluoresce em cerca de 30\% (Kaplan et al. ${ }^{18}, 1958$ ), 31,6\% (Carman et al. ${ }^{\circ}$, 1979), 44\% (Pepin: Ausiwick ${ }^{32}$, 1968), 50\% (Muller et al. ${ }^{31}$. 1983) ou 64,5\% dos casos de microsporíase dos cães e gatos (Dubugras êt al. ${ }^{13}, 1922$ ). Neste casos, em particular, também não se detectou fluorescência. Todavia, para que se alije completamente este exame da estratégia diagnostica é preciso que se colecione um número maior de casos para que se obtenha a necessária representatividade amostral.

\section{AGRADECIMENTOS}

É mister que se agradeça aos colegas médicos veterinários Sebastião de Almeida, Eduardo Lopes Eziliano, André T. Carrascoza, Jorge Teivellis Filho, Thomas Walter Wolff, Luis Alberto Lopes, Luis Alberto Marinho, Ruy Carlos Vincenzi, que permitiram o acesso aos eqüinos com quadro clínico compatível com dermatofitose.

\section{SUMMARY}

Due to the scarcity of reports, in Latin-American and Brazilian literature, related to fungal microbiota of healthy horses, or to the main species of dermatophytes in horses showing suggestive lesions of dermatophytic infections, 175 horses of both sexes and different ages, including purebred horses, crossbreed, or others with no specified breed were divided in 2 groups. Group 1 was constituted by 133 asymptomatic horses without any cutaneous lesions; after dermatologic examinations, horses were submitted to Wood's Light (48 horses), samples taken by "carpets technique" and later cultivated in Agar Sabouraud Dextrose, Mycobiotic Agar, Trichophyton Agar 3, Trichophyton Agar 5, at temperature of $25^{\circ} \mathrm{C}$ and $37^{\circ} \mathrm{C}$ during 30 days. The fungal species isolated were Penicillium sp (80.4\%), Rhizopus sp (62.4\%), Aspergillus sp (41.3\%), Fusarium sp (40.6\%), Cladosporium sp (33.1\%), Trichoderma sp (21.0\%), Mucor sp (18.0\%), Epicoccum sp (12.0\%), Mycelia sterillia (8.8\%), Rhodotorula sp (7.5\%), Neurospora sp (4.5\%), Alternaria sp (3.7\%), Aureobasidium sp (3.7\%), Geotrichum sp (3.0\%), Paecilomyces sp (2.2\%), Monascus sp (2.2\%), Cephalosporium sp $(1.5 \%)$, Nigrospora sp (0.7\%), Scopulariopsis brevicaulis $(0.7 \%)$ and Trichosporon sp (0.7\%). Group 2 was represented by 42 horses presenting suggestive lesions of dermatophytosis, and after being submitted to dermatologic examination and to Wood's Light (22 horses), hairs and scales or crusts were then cultured. From these, 6 (14.3\%) horses were positive for fungal growth identified as Dermatophylus congolensis, and from $3(7.1 \%)$ other horses, Microsporum canis was isolated. Of the 70 equines exposed to Wood's Light, false fluorescence was found in 2 horses.

UNITERMS: Dermatophytes; Equidae. 
ISHIKAWA. M.M.; LUCAS. R.: LARSSON. C.E.; GAMBALE. W.: FERNANDES, W.R. Isolamento e identificação da microbiota fúngica e de dermatófitos da pele de eqüinos hígidos e ditgueles afetados por dermatofitone. Braz. J. vet. Res. anim. Sci. São Paulo, v.33, n.3. p. 170-175, 1996.

\section{REFERÊNCIAS BIBLIOGRÁFICAS}

1-AHO. R. Saprophytic fungi isolated from the hair of domestic and liboratory animals with suspected dermitophytosis. Mycopathologia, v.83, p.65-73. 1983.

2-AINSWORTH, G.C.: AUSTWICK, P.K.C.A. Survey of animal mycoses in Brilain: general ispects. Veterinary Record, v.67, p.88-97, 195.

3-ARX, Y.V. Fungi sporulation in pure cultures. London, I. Cramer, 1970.

4.BARBOSA, M.: MOREIRA. E.C.: MOREIRA, Y.K.; FONSECA I.C. Dematofilose en equiinos no Estado de Minas Gerais. Brasil. Arquivos da Iscola de Veterinária da I niversidade Federal de Minas Gerais, v.22, $1.227-9,1970$

5-BARRON, (i.L. The genera of Hyphomycetes from soil. New York, Willians an Wilkins, 1971

6-BODIN. E.. I896 apud GEORG, L.K.; KAPLAN, W.: CAMP, V.B. Equine ringworm will specific reference 10 Tricophyion equinum. American Journal Veterinary Research, v. 18, p.798, 1957

7-BROCQ-ROSSEU. D.: URBAIN. A.: BAROTTE. J. Étude des teignes du cheval el immunité daus les leignes experimentales. Annales de l'Instifut Pasteur, Paris, v.41, p.513-51, 1927

8-CARMAN. M.G.: RUSH-MUNRO, F.M.: CARTER, M.E. Dermatophytes isolated trom domestic and feral animal. New Zealand Veterinary Journal, v.27, p. 136,1979

9-CARTER, G.R.: BENEKE, E.S.: MCALLISTER, H.A. Ringworm of horse calused by an atypical form of $M$. cemis. Journal of the American Veterinary Merlical Association, v. 156, p. 1048-50, 1970.

10-CHENGAPPA. G.: MADDUX. R.L.: GREER, S.C.: PINCUS. D.H.; GEIST, L.L. Isolation and identilication of yeasts and yeast-like organisms from clinical veterinary sources. Journal Clinical of Microbiology, v. 19, p.427, 1984.

11-COLES, B.M.. 1978 apud SCOTT, D.W. v.45. p. 174

I2-DAVIS, C.L.; SHAEFER. W.B. Cutaneous aspergillosis in a cow. Journal of the American Veterinary Medical Association, v. 145, p.692, 1962.

13-DUBUGRAS, M.T.B.: LARSSON, C.E.; LEDON, A.L.B.P.; GAMBALE, W. Dermatofitoses e leveduroses de cães e gatos. Aspectos diagnósticos. Brazilian Journal of Veterinary Research and Animal Science. São Paulo, v.29, 11.2, p.273-87, 1992.

14-ENGLISH. M.P. The saprophytic growth of non-keratinophylic fungi on keratinized substracta in compatrison with keratinophilic lungi. Transactions of the British Mycological Society, v.48, p.219-35, 1965.

15-EUZEBY, J.: LACLAINE, C.: CHAVVE, C .; EGHBALI, A. Investigations mycologiques I. Recherches sur quelques pseudo-dematophytes. Bulletin de la Société des Sciences Vétérinaires et de Médecine Comparée de Lyon, v.75, p. 355-60), 1973.

16-FISCIIMAN. O. Ringworm infection by Microsporum canis in a horse. Mycopathologia et Mycologia Applicata, v.30, p.273, 1966.

17-GAMBALE, W.; CORREA, B.; PAULA, C.R.; PURCHIO. A.; LARSSON. C.E. Ocorrência de fungos em lesöes superficiais de cães na cidade de São Paulo. Revista da Faculdade de Medicina Veterinária e Zootecnia da U/niversidade de Sáo Paulo, v.24, 11.2, p. 187-9), 1987.

I8-KAPLAN, W.: GEOR(i, L.K.: AJELLO). L. Recent developments in animal ringworm and their public heallh implications. Annals of the New York Academy of Sciences, v. $70,0.636-49,1958$

19-KREGER-VON RIJ. N.J.W. The yeasts: a taxonomic study. 3.ed. Amsterdam. Elsevier, 1984

20.LINCON, S.P): ADCOCK. J.1. Disseminaled geotrichosis in al dog. Pathology Veterinary, v.5, p.282-9, 1968

2 l-LODDER, Y. The yeasis: a taxonomic sludy. 2.ed. Amsterdam, North Hollind, 1970

22-LONDERO. A.T. An epizootic of Trikhlorfon equinmm infection in horses in Brazil. Sabouraudia, v. 3, p. $14,196.3$

23-LONDERO, A.T. Dermallophilus infection in the subtropical zone of South Americal. In: LLOYD, D.H.: SELLERS, K.C., ed. Dermatophilus infection in animals and man. London, Academic Press, 1976. p.110-5.

24-LONDERO, A.T.; RAMOS, C.D.; LOPES, J.S. A ten survey of the cutaneous mycoses in the state of Rio Grande do Sul, Brasil. Revista do Instituto de Medicina Tropical de Sāo Paulo, v.12. p.339-42, 1970.
25-LONDERO, A.T.; SANTIAGO. M.: RAMOS, C.D. Dermatile micólicit em eqüinno. Revista de Medicina Veterinária, São Paulo, v.3, p.77, 1967

26-McGINNIS, M,R. Laboratory handbook of medical mycology. New York, Acildemic Press. 1980.

27-MARIAT, F.; ADAN-CAMPOS, C. Lal technicue du carré de lapis mélhode simples de prévelement dans les mycoses superlicielles. Annales de I'Institut Pasteur, v.113, p.666-8. 1967

28-MARIAT, F.: TAPIA, G. Dénobrennent des champignons keratinophiles d'une population de cynocéphales (Papio papio). Annales de Parasitologie, Paris, v.41. n.6. p.627-34. 1966

29-MORQUER, R.; LOMBARD, C.; BERTHELON, M. Pouvoir pathogène de quelques especes de geotrichum. Comptes Rendus Hebdomadaires des Seances de I'Academie des Sciences, Paris, v.240, p.378-82. 1955.

3()-MOS, E.N.: MACRUZ, R.: SANTOS, M.R.S.; PORTO, E. Georricose em equiino Puro Sangue Inglês. Revista da Faculdade de Medicina Veterinária de São Paulo, v. 15, p.93-8. 1978.

31-MULLER, G.H.; KIRK. R.W.: SCOTT, D.W. Simall animal dermatology. 3.ed. Philadelphia, W.B. Situnders, 1983

32-PEPIN, G.A.: AUSTWICK. P.K.C. Skin Disease, mycological origin. Veterinary Record, v.82, p.208-14, 1968

33-PERCEBOIS, G.; BIAVEL, M.F.; KURES, L. Discussion du pouvoir pathogène de ecrtanes especes d'Alternariat a propos de olres observations. Bulletin de la Société Francaise de Mycologie el Médecine, v.7, p.15-8, 1978.

34-PEREZ-CATAN. E.; DI ROCCO. M. Dermatitis contagiosa a Demmatophilus (ongelensis en equinos. Revista de Investigacion Agropecuaria, Buenos Aires, v.4, n.2, p.161, 1965

35-PORTUGAL, M.A.S.C.: FISCHMAN. O. Culaneons Streptothricosis in al mule. Mycopathologia et Mycologia Applicata, n.3, p.197-200, 1974

36-PORTUGAL, M.A.S.C.: GIORGI, W.: SALLES GOMES, C.E.; SALLES GOMES, T.L. Primeiras observações sobre ocorrência da dermatofitose em equüinos no Estado de São Paulo. Biológico, v.45, p.299-304, 1979.

37-PROENCA, N.G.: ASSU MPCÃO, S.B.P. Dermatofitoses observadas em crianças com 12 anos de idade, em Săo Paulo. Revista do Instituto de Medicina Tropical de Sáo Paulo, v.21, p. 146-8, 1979.

38-RAPER, K.B.; FEWELL. D.I. The genus Aspergillus. Baltimore, Williant and Wilkins. 1965.

39-REBELL, G.; TAPLIN, D. Dermatophyles: Their recognition and identification, Miami, University of Miami Press, 1970

40-SANTOS: M.R.S.; CAMARGO), Z.P. Classilicação de levedurats de processos inflamatórios genitais de equimos Puro Sangue Ingless. Biológico, São Paulo, v.4. p.162-7, 1975.

4I-SANTOS, M.R.S.: PORTUGAL. M.A.S.C.: FERRAZ, E.F. Pesquisa da presença de fungos derınatófitos no tegumento de animais alojados no. Jockey Club de Sáo Paulo (1969-1988). Säo Paulo, 1992 (Informação pessoal).

42-SANTOS. M.R.S.: PORTUGAL, M.A.S.C.: ATHAIDE, A.J.; FERRAZ. E.F. Gcotricose cutânea em eqüinos. In: CONGRESSO BRASILEIRO DE MEDICINA VETERINARIA, 13, Brasília, 1972. Anais, p.30).

43-SANTOS. M.R.S.: PORTUGAL. M.A.S.C.: ATHAIIDE. A.J.: FERRAZ. IE.F. Gcotricose cutânea em equiinos. Biológico. São Paulo, v.49, p.75-9. 1983.

44-SCOTT, B.W.; BENTINCK-SMITH, I. Cultures performed on hair and skin scrapings from lurec diferent areals from each of four normal horses, 1985. apud SCOTT, D.W.45, p.542.

45-SCOTT. D.W. Foliculitis and furunculosis. In: ROBINSON. N.E. Current therapy in equine medicine. Philadelphia. W.B. Satunders. 1985. p.542.

46-SCOTT, D.W. Large animal dermatology. Philadelphia, W.B. Sianders, I988.

47-TABUCHI, K. Papular dermalitis in pigs. Therapeutic trials against Aspergillus. Bulletin of the Arabu Veterinary College, v. II. p.67. 1963.

48-TAKATORI. K ; ICHIJO, S.; KONISHI. T; TANAKA, 1. Ocorrence ol equine dermalophylosis in Hokkaido. Japamese Journal of Veterinary Science, v.43, p.307, 1981 .

49-YLIMAKI. A. The mycoflora of cereal seeds and some feed stufts. Annale de Agriculture Female, v.20, p.74-88, 1981.

Recebido para publicação: 27/01/94

Aprovado para publicação: 08/11/95 